\title{
Solitary bone metastasis to the tibia from colorectal cancer- A case report
}

\author{
Abdulsalam Alnajjar, Asit Kumar Mohanty \\ Department of Medical oncology, Kuwait Cancer Control Centre, Safat, Kuwait.
}

Received June 15, 2014; Revised July 24, 2014; Accepted July 26, 2014; Published Online September 08, 2014

\section{Case Report}

\begin{abstract}
The onset of osseous metastases during the course of colorectal cancer is not common. Although rare, they usually appear in the axial skeleton. In our report, we refer to the case of a 48-year-old patient who presented with colon cancer and eventually developed a solitary bone metastasis in the upper end of left tibia. At the time of diagnosis and staging investigations, the patient had only a primary disease.
\end{abstract}

Keywords: Solitary Bone Metastasis; Tibia; Colorectal Cancer; Chemotherapy; Radiotherapy

\section{Introduction}

A 48 -year-old woman presented in January 2012 with features of acute intestinal obstruction. Computer tomography scan revealed circumferential thickening of a long segment of bowel loop involving the descending colon with a narrowed lumen. Left hemicolectomy with Hartman procedure was performed and the pathological examination revealed adenocarcinoma colon with a pathological TNM staging of pT3N3Mx. The patient received adjuvant chemotherapy with Capacitabine and Oxaliplatin for 4cycles. Serum Carcino Embryonic antigen level before the surgery was $5.8 \mathrm{ng} / \mathrm{ml}$. A follow up CT scan after 4 cycles of chemotherapy in June 2012 revealed bilateral ovarian lesion with solid and cystic components. She underwent bilateral salpingo-oopherectomy and histopathology revealed metastatic adenocarcinoma, consistent with the primary tumor. She was continued on adjuvant combination chemotherapy comprising of Capacitabine, Oxaliplatin and Bevacizumab.

She was doing well and was asymptomatic for about two months, in August 2012 the patient presented with a painful enlargement in her left tibia. On clinical examination there

Corresponding author: Abdulsalam Alnajjar; Department of Medical oncology, Kuwait cancer control centre, Safat, Kuwait.

Cite this article as:

Alnajjar A, Mohanty AK. Solitary bone metastasis to the tibia from colorectal cancer- A case report. Int J Cancer Ther Oncol 2014; 2(4):02045. DOI: 10.14319/ijcto.0204.5 was a palpable, tender lesion in the upper end of her left tibia measuring $8 \times 4 \mathrm{~cm}$. The patient did not complain for any other symptoms and the rest of the clinical examinations were unremarkable. A 99mTc-MDP bone scintigram showed an abnormal uptake in the left tibia, revealing an osseous solitary metastasis Figure 1. In MRI radiography a single osteolytic lesion was seen in the diaphysis of the left tibia covering almost all the diameter of the bone in this site Figure 2. The patient underwent biopsy on her left tibia which showed bone metastasis of colonic origin. A new chest/abdominal/pelvic CT scan revealed neither any other visceral metastases nor a local recurrence.

In August 2012, external beam radiotherapy was given to her left tibia using a 2-field technique, delivering $8 \mathrm{~Gy}$ in one single fraction by a $\mathrm{Co}^{60}$ unit, managing to relieve the patient's symptoms. To delay clinical progression of the tumor the patient continued her chemotherapy along with injections of Zoledronic acid (4 mg every 3 week). She tolerated her treatment well with no significant side effects. She underwent internal fixation of the tibia for an impending fracture in November 2012.

A recent complete CT scan of the whole body was negative for other metastases. The patient is receiving palliative chemotherapy with tablet Capecitabine $\left(20000 \mathrm{mg} / \mathrm{m}^{2} \mathrm{~d} 1-14\right)$ along with Oxaliplatin $\left(130 \mathrm{mg} / \mathrm{m}^{2} \mathrm{~d} 1\right)$, Bevacizumab $(5 \mathrm{mg} / \mathrm{kg})$, and injection Zoledronic acid. She is doing well with no significant side effects. She remains in partial remission with periodic CT scans at every 3 months showing no 
new lesions. The lesion in her left tibia remains visible in plain X-ray and continues to show uptake in the bone scan 6 months after commencing chemotherapy now. Her ECOG performance status is 2 with mild to moderate pain requiring painkillers occasionally. The CEA level has dropped back to normal.

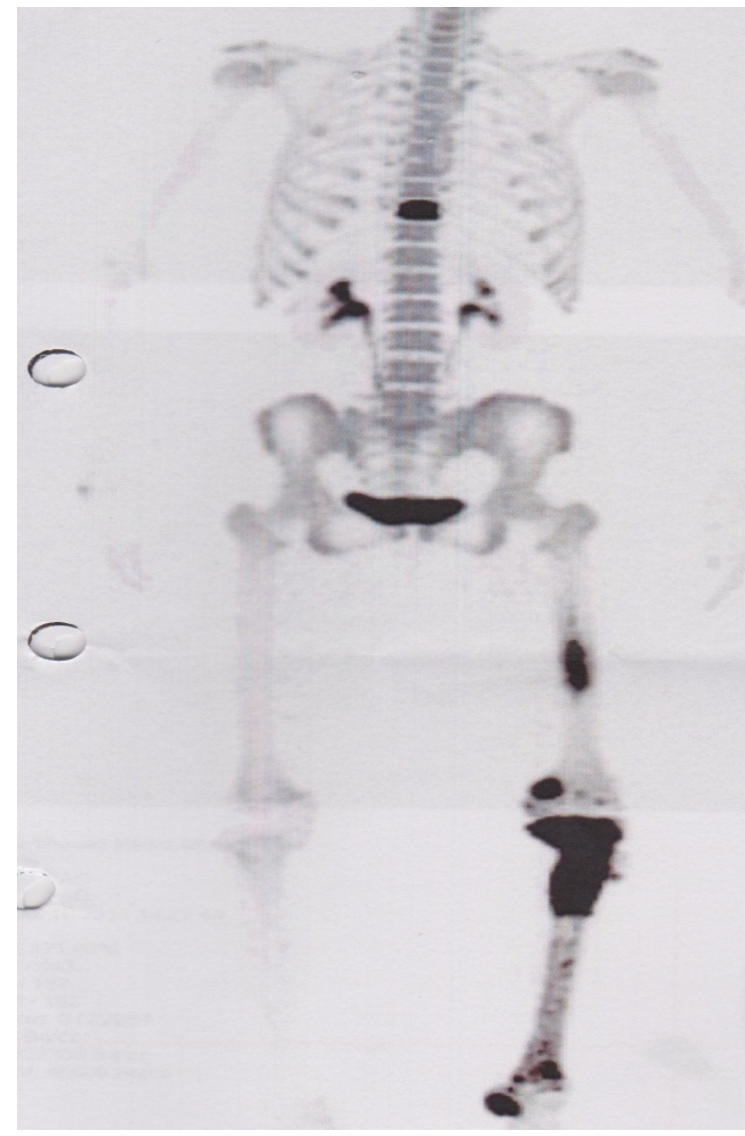

FIG. 1: 99Tc-MDP bone scintigram showing abnormal uptake in the left tibia.

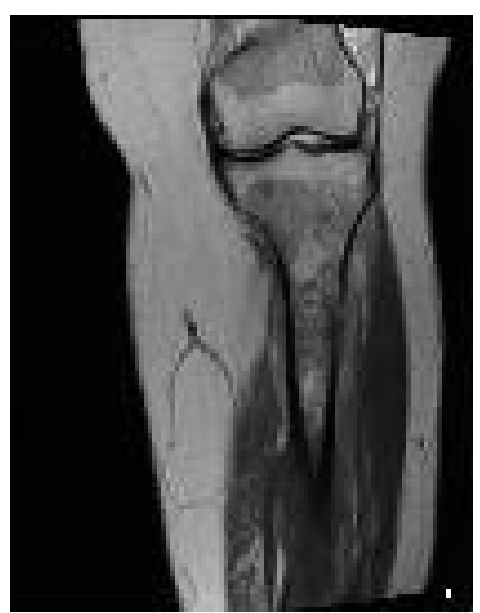

FIG. 2: In MRI radiography a single osteolytic lesion could be seen in the diaphyses of the left tibia.

\section{Discussion}

Death from colorectal cancer is usually attributed to progression of the disease with recurrence and metastases. Skeletal metastases in the primary colorectal cancer are an uncommon event. The incidence of bone metastasis is reported in the English literature to be between 4.7 and $10.9 \%$ in clinical cases and up to $23.7 \%$ in autopsy cases. ${ }^{1}$ In the majority of cases they are associated with liver or lung cancers. Solitary skeletal metastases from a primary colonic carcinoma are a rare event, with a very low incidence of $1.1 \%$ in a retrospective review from Kanthan et al. ${ }^{2}$ The site of the osseous metastasis in our case is also rare, with only 5 cases reported in the literature previously, one from Decker and Fash ${ }^{3}$, second case reported by Creedon ${ }^{4}$, and 3 others cases noticed by Kanthan et al. ${ }^{2}$

There is a difference in the frequency of bone metastases between colon and rectal cancer. As Masaru et al. ${ }^{1}$ have demonstrated in their autopsy series, rectum cancers are more frequently associated with bone metastases than cancers from other portions of the large gut. Also comparing between various histological types, signet ring cell carcinoma shows a high incidence of bone metastasis. ${ }^{1,5,6}$

The most common sites of osseous metastases are the vertebrae, pelvis and sacrum. Osseous metastases in these sites are blood-borne through veins, and the paravertebral venous plexus of Batson. ${ }^{7}$ The direct spread of malignant cells from prostatic, mammary, and gastrointestinal cancers to the vertebral venous plexus of Batson explains the high frequency of vertebral bony metastases in these primary tumors. The communications between the spinal veins in the lumbar region with the iliofemoral venous system may indicate that colonic and rectal neoplasms as well as vesical tumors seed to the lower extremities in a retrograde fashion. ${ }^{8}$

Disease-free survival from the time of diagnosis to the onset of skeletal metastases ranges from 10 to 5,309 days according to Kanthan et al. ${ }^{2}$ They also concluded that $38 \%$ of patients with bone only vs. $16 \%$ with bone and visceral metastasis were alive at 5 years follow-up, although no statistical difference was found in the 10-year survival rate between the two groups. Also Nozue et al. ${ }^{6}$ reported a median interval of 16.5 months from the initial diagnosis and operation to the onset of osseous metastases (range 0-108 months). The 1-year survival rate was $20 \%$ in the above series and the patients with solitary osseous metastasis (2 patients) survived for more than 1 year.

For the early detection of osseous metastases bone scan is considered the most effective method. In plain radiography the lesions may be osteolytic or osteoblastic (less common). Talbot et al. ${ }^{9}$ found that patients with symptomatic bone metastases were diagnosed at a median time of 21 months after initial surgical excision of the primary tumor. Our pa- 
tient was diagnosed to have bone metastasis after 8 months of completion of surgery.

Although solitary bone metastases from colorectal cancers are rare, the evidence from the literature shows that we should consider osseous metastasis as a cause of bone pain in patients with colorectal cancer. These patients, as has previously been reported, have a better prognosis than patients with multiple bone metastasis and palliative chemotherapy and radiotherapy should be offered to them if they are eligible.

\section{Conflict of interest}

The authors declare that they have no conflicts of interest. The authors alone are responsible for the content and writing of the paper.

\section{References}

1. Katoh M, Unakami M, Hara M, Fukuchi S. Bone metastasis from colorectal cancer in autopsy cases. $J$ Gastroenterol 1995; 30:615-8.

2. Kanthan R, Loewy J, Kanthan SC. Skeletal metastases in colorectal carcinomas: a Saskatchewan profile. Dis Colon Rectum 1999; 42:1592-7.

3. Decker FH, Fash JC. Carcinoma of rectum with metastasis to tibia. Med Radiogr 1950; 26:125-8.

4. Creedon F. Solitary peripheral bone metastasis from carcinoma of the rectum. Br J Surg 1966; 53:999-1001.

5. Besbeas S, Stearns MW Jr. Osseous metastasis from carcinoma of the colon and rectum. Dis Colon Rectum 1978; 21:266-8.

6. Nozue M, Oshiro Y, Kurata M, et al. Treatment and prognosis in colorectal cancer patients with bone metastasis. Oncol Rep 2002; 9:109-12.

7. Batson OV. The function of the vertebral veins and their role in the spread of metastases. Ann Surg 1940; 112:138-49.

8. Libson E, Bloom RA, Husband JE, Stoker DJ. Metastatic tumours of bones of the hand and foot. Skeletal Radiol 1987; 16:387-92.

9. Talbot RW, Irvine B, Jass JR, et al. Bone metastases in carcinoma of the rectum: a clinical and pathological review. Eur J Surg Oncol 1989; 15:449-52. 